\title{
A Quiescent, Regeneration-Responsive Tissue Engineered Mesenchymal Stem Cell Bone Marrow Niche Model via Magnetic Levitation
}

Emily Elizabeth Louise Lewis, ${ }^{\dagger}$ Helen Wheadon, ${ }^{\ddagger}$ Natasha Lewis, ${ }^{\dagger}$ Jingli Yang, ${ }^{\dagger}$ Margaret Mullin, ${ }^{\S}$ Andrew Hursthouse, "David Stirling," Matthew John Dalby, ${ }^{\dagger}$ and Catherine Cecilia Berry ${ }^{*}{ }^{\dagger}$

${ }^{\dagger}$ Centre for Cell Engineering; Institute of Molecular, Cell, and Systems Biology (IMCSB) and ${ }^{\S}$ Electron Microscopy Unit, University of Glasgow, Joseph Black Building, University Avenue, Glasgow G12 8QQ United Kingdom

${ }^{\ddagger}$ Paul O’Gorman Leukaemia Research Centre, University of Glasgow, Shelley Road, Glasgow G11 0YN, United Kingdom

"School of Science, University of the West of Scotland, Paisley PA1 2BE, United Kingdom

\section{Supporting Information}

ABSTRACT: The bone marrow niche represents a specialized environment that regulates mesenchymal stem cell quiescence and self-renewal, yet fosters stem cell migration and differentiation upon demand. An in vitro model that embodies these features would open up the ability to perform detailed study of stem cell behavior. In this paper we present a simple bone marrow-like niche model, which comprises of nanomagnetically levitated stem cells cultured as multicellular spheroids within a type I collagen gel. The stem cells maintained are nestin positive and remain quiescent until regenerative demand is placed upon them. In response to coculture wounding, they migrate and appropriately differentiate upon engraftment. This tissue engineered regeneration-responsive bone marrow-like niche model will allow for greater understanding of stem cell response to injury and also facilitate as a testing platform for drug candidates in a multiwell plate format.

KEYWORDS: mesenchymal stem cells, bone marrow niche, magnetic nanoparticles, tissue engineering, migration, scratch assay, wound homing, differentiation

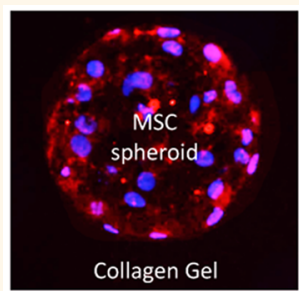

Quiescent MSC spheroids, via magnetic levitation, cultured in a collagen gel act as bone marrow nichelike models.

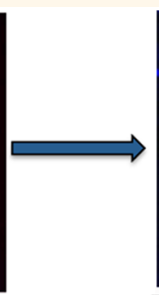

Paracrine signaling in
response to co-cultured induced MSC migration and cell type specific regeneration

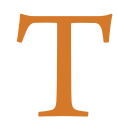
he bone marrow is resident to multipotent mesenchymal stem cells (MSCs), which are localized in a niche environment regulating MSC quiescence, selfrenewal, and differentiation. ${ }^{1,2}$ Aside from the benefits of multipotency, MSCs home to sites of cellular injury by migrating from the niche to the injured tissue on regenerative demand; these attributes make them key targets for potential clinical use and make modeling the niche to allow deeper understanding a major research drive., ${ }^{3,4}$ In this report, we present an in vitro niche model that is not only simple and quick to assemble but also mimics the MSC niche with the capacity to support viable, nestin+, quiescent MSCs within a low stiffness collagen I scaffold simulating bone marrow. The use of superparamagnetic nanoparticles (SPION) to achieve magnetic levitation allows us to generate multicellular MSC spheroids within a few hours rather than days as required by other established methods, e.g., hanging drop. However, and critically, the use of SPION allows ease of cell tracking, and thus we demonstrate that our nanoniches can respond to regenerative demand through cell migration, engraftment, and 

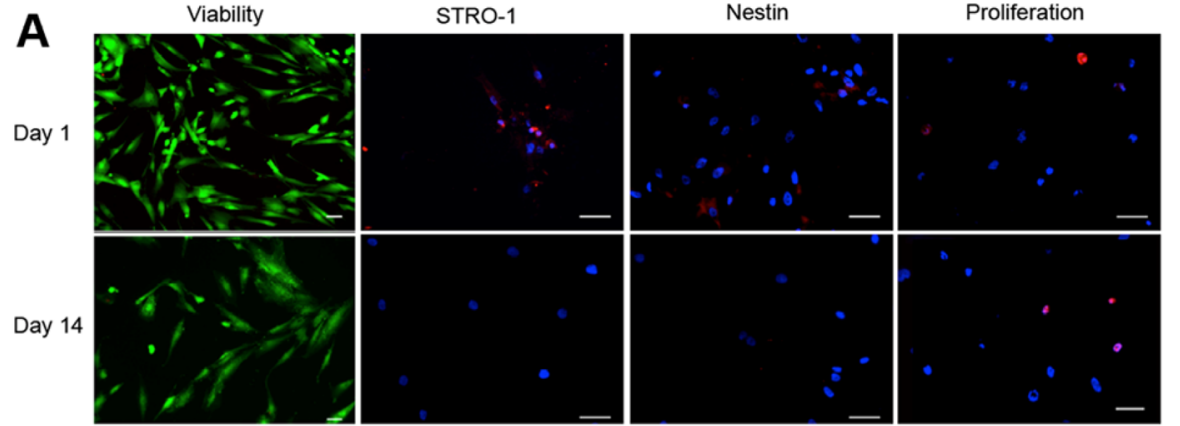

Monolayer

culture
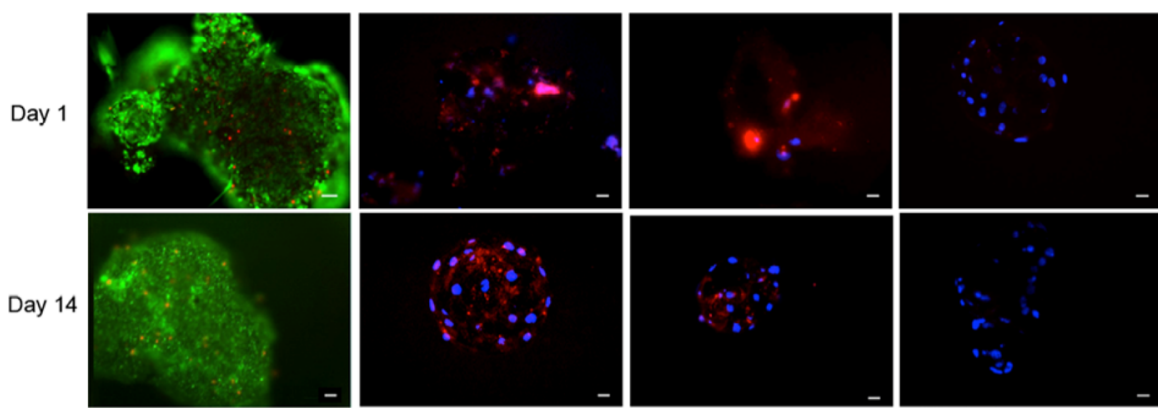

Spheroid

culture
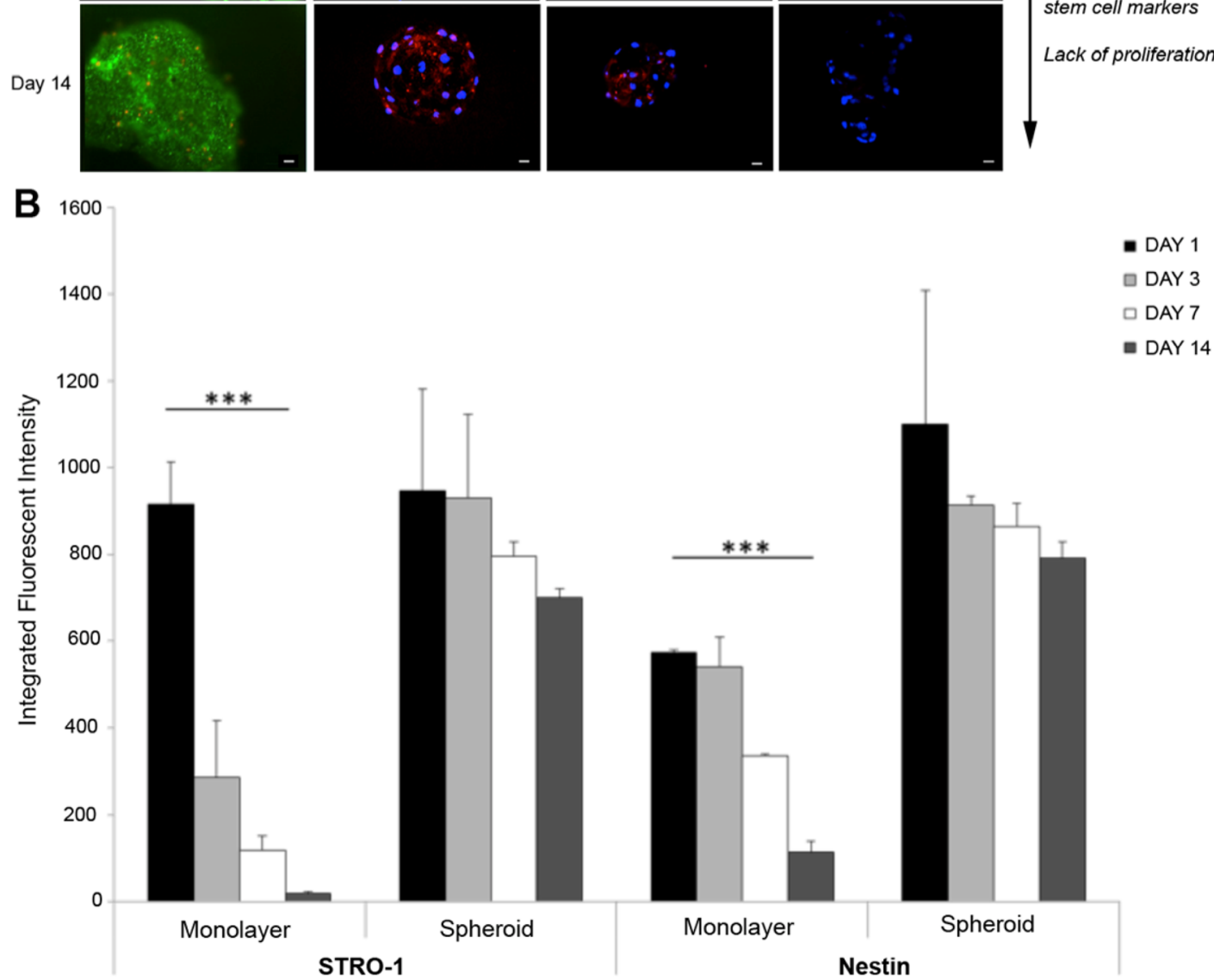

Figure 1. Monolayer and spheroid timeline culture of mNP labeled MSCs. (A) Images were taken at days 1 and 14 within media assessing; vertical panel 1 cell viability (green $=$ living cells, red $=$ dead cells, $10 \times$ objective), panel 2 and 3 phenotype $($ red $=S T R O-1 /$ nestin, blue $=$ nucleus, $20 \times$ objective) and panel 4 proliferation ( $\mathrm{red}=$ proliferating, blue $=$ nuclei). Scale bar $=\mathbf{5 0} \mu \mathrm{m}$. Note the decreasing MSC phenotype with time in monolayer, with increasing cell division and growth, while MSC phenotype is maintained in spheroid culture, with cessation of cell division. (B) Graph indicates quantitative analysis of STRO-1 and nestin in monolayer and spheroid culture at days $1,3,5$, and 14 ( $n=3$ samples; 6 image from each). (Error bars denote standard deviation; * indicates statistical significance where $p<0.05$, ** indicates $p<0.01$ and $* * *$ indicates $p<0.001$ as calculated using one way ANOVA.) Note progressive loss of marker in monolayer with retention in spheroid culture.

methodology that speeds up niche formation and develops this further to introduce a $3 \mathrm{D}$ extracellular matrix environment. The bone marrow is a low-stiffness collagenous matrix, and it is notable that MSCs cultured in low-stiffness biomaterials express cell surface markers such as nestin. ${ }^{12-14}$ Nestin, normally considered a neural marker, is now recognized as being key in cell-cell interaction, motility, and signaling within the niche; within the marrow, hematopoietic stem cell (HSC) home to resident nestin+ MSCs. It is postulated that expression of such proteins may be due to the tendency of adult stem cell niches to be associated with adrenergic nerve fibers linking niches to circadian regulation. ${ }^{15}$ If MSCs do not express nestin in the niche, then HSCs mobilize to the spleen. ${ }^{16}$ 

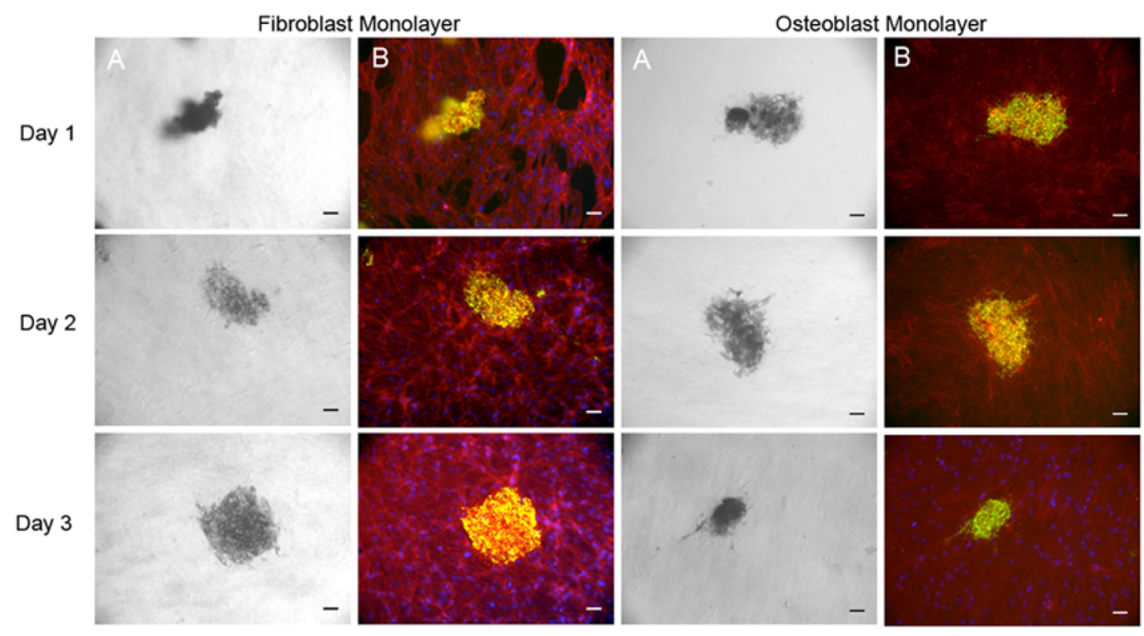

Intact monolyaer

(i.e. non-activated)
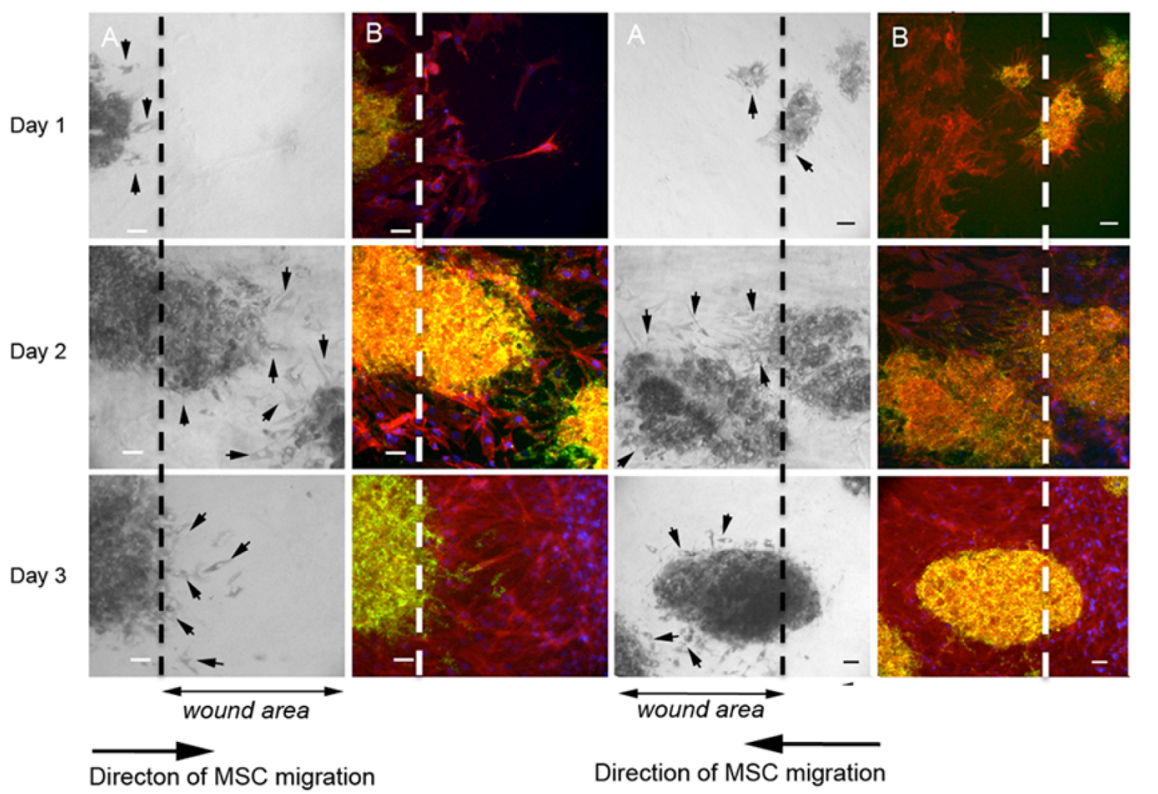

Scratched

monolyaer (i.e. activated)

No MSC migration from spheroid

MSC migration out of spheroid towards wound

Figure 2. Control (upper) and activated (lower) fibroblast and osteoblasts monolayer wound healing assay, with spheroid coculture above. MSC spheroids cultured in a type I collagen gel directly over both an intact fibroblast and primary osteoblast monolayer (upper panel) and a scratched (activated) monolayer (lower panel); dashed line indicates wound edge. (A) Light microscopy images of spheroids; (B) immunofluorescent images of spheroids and monolayer cells $($ red $=$ actin, green $=\mathbf{m N P s}$, blue $=$ nucleus). $10 \times$ objective, scale bar $=50 \mu \mathrm{m}$. Note the lack of MSC migration in control cultures, with MSC migration from the spheroid toward the wound in activated cultures. Arrowheads denote examples of migrations cells (labeled green with NPs in fluorescent image).

\section{RESULTS AND DISCUSSION}

MSC Spheroids are Viable, Quiescent and Express STRO-1 and Nestin. To produce our MSC spheroids, primary human bone marrow MSCs were initially labeled with fluorescent magnetic nanoparticles (Supplementary Figures $1-4$ ) in monolayer, with the particles being retained within the cells post-trypsinization (Supplementary Figure 4). Once labeled, the cells were retrypsinized and levitated in cell media within a 24-well plate via an external magnet above the plate; this coerces the cells together to form a multicellular spheroid via "magnetic levitation". The magnetic field has been previously characterized and is sufficient to permit spheroid generation at the media/air liquid interface. ${ }^{17,18}$ Aside from the original cell seeding density, spheroid generation via magnetic levitation does not permit exact control over spheroid size and shape, however spheroids form with a mean diameter of 259 $\mu \mathrm{m}$ (Supplementary Figure 5). After formation $(\sim 3 \mathrm{~h})$, the external magnets were removed, and the spheroids were subsequently transplanted into a soft type I collagen gel (Supplementary Figure 6), with a modulus akin to bone marrow (Supplementary Figure 7; 40 Pa Young's Modulus). The MSCs were viable, maintained high expression of the stem cell markers STRO-1 and nestin, and were quiescent within their magnetic spheroids for up to day 14, as compared to standard cultures in monolayer where MSCs proliferated and stem cell markers were lost by day 3 of culture (Figure 1). Furthermore, the MSCs cultured in spheroids remained multipotent and were capable of differentiation into both osteoblasts, adipocytes, with expression of chondrogenic transcription markers after spheroid dissociation and culture in monolayer (Supplementary Figures 8, 9) and secreted increasing levels of CXCL12 with time in culture (Supplementary Figure 10).

MSCs Transmigrate from the Spheroid Niche Toward Cocultured Wound Sites. With a view to assessing the MSCs potential to home and engraft from the niche model to sites of 


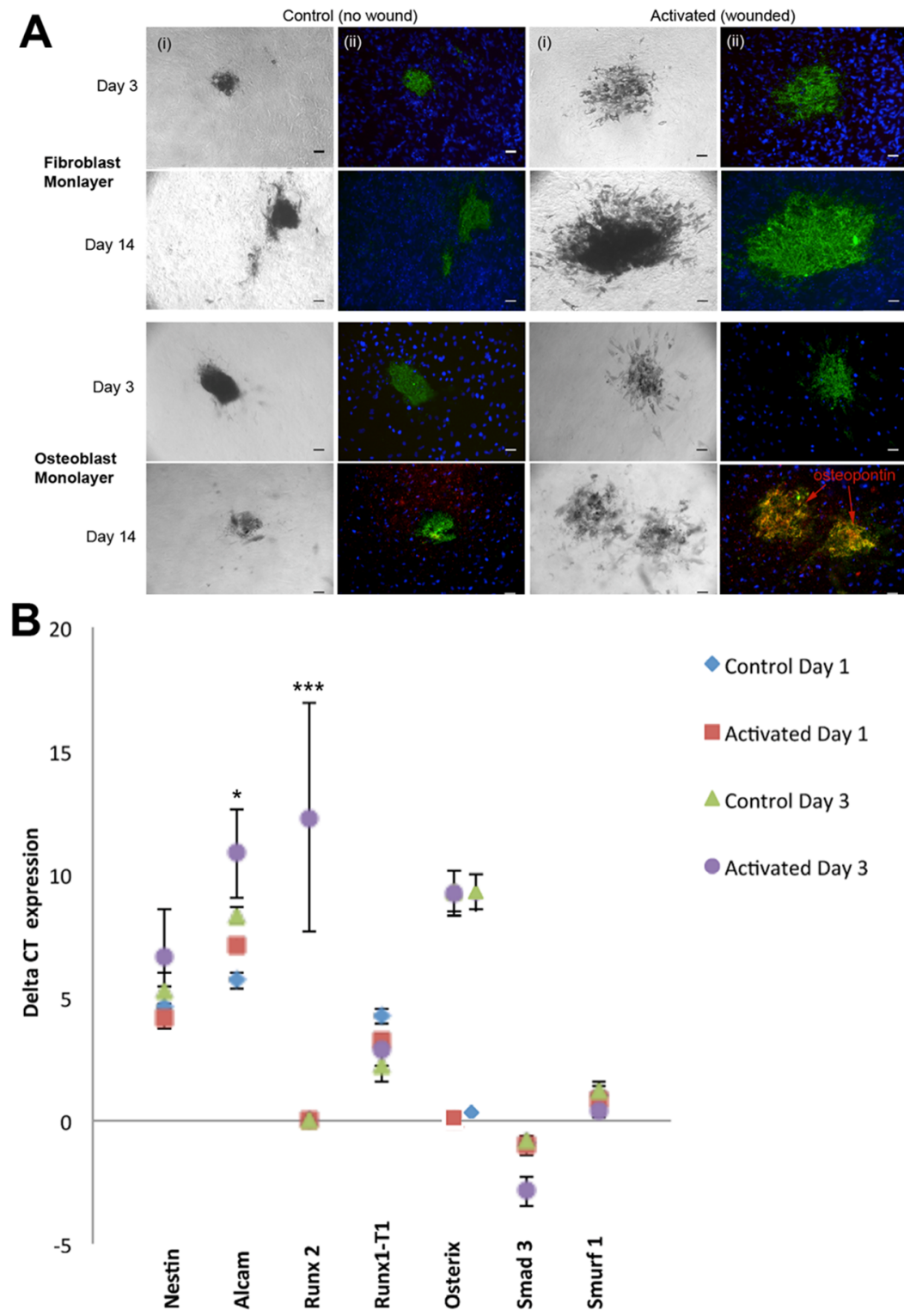

Figure 3. MSC osteoblastic differentiation during wound healing. (A) MSC migration and osteoblast differentiation assessed at days 3 and 14 of culture, in control and activated wound models. (i) Light microscopy image of spheroids; (ii) immunofluorescent images of spheroids and monolayer cells $[$ red $=$ phosphorylated runx $2($ day 3$) /$ osteopontin $($ day 14$)$, green $=$ mNPs, blue $=$ nucleus]. $10 \times$ objective, scale bar $=50 \mu \mathrm{m}$. MSC migration from the spheroids was noted in both fibroblast and osteoblast activated models. Phospho runx 2 staining was also picked up at day 3 (Supplementary Figure 9). Arrowheads denote osteopontin staining in MSC spheroid at day 14. (B) MSC and osteoblastic differentiation markers during control and activated osteoblast wound healing model [days 1 and 3; graph represents $n=3$ (pooled from $n=$ $6)$, mean \pm SD; stats indicate one-way ANOVA significance (*) of activated compared to corresponding control]. MSC spheroid RNA was harvested and analyzed via fluidigm on days 1 and 3 of coculture with both the control and activated osteoblast wound models. Note maintenance of MSC markers under all conditions, but increased osteoblastic markers under activated cultures, in particular the high runx2 expression in the activated day 3 model.

injury, the MSC spheroids were cocultured with a simple wound healing model. Briefly, this entailed either human fibroblasts or osteoblasts being cultured in multiwell plates in monolayer and scratched with a pipet tip to create a wound (Supplementary Figure 11), and the spheroids contained within collagen gels being immediately overlaid. MSC response was initially gauged over 3 days of culture. When the spheroids were cocultured over intact fibroblast and osteoblast monolayers (i.e., unscratched controls), the MSCs remained within the spheroids (Figure 2). However, when cocultured over wounded fibroblast and osteoblast monolayers (i.e., activated cultures in need of regeneration), the MSCs migrated out of the spheroids toward the wound, appearing to incorporate and engraft into the underlying monolayer (Figure 2). This MSC migration and incorporation into the fibroblast and osteoblast monolayers closely reflect the in vivo situation of MSC homing and engraftment in response to injured tissues.

Following Transmigration, MSCs Differentiate Appropriately to Assist Tissue Regeneration. We subsequently determined whether the MSCs were changing phenotype during transmigration toward the wound. This was assessed by repeating the MSC spheroid coculture with the fibroblast, osteoblast, and additionally chondrocyte wound models. Osteoblast differentiation analysis of early and late osteoblast 
phenotype markers, phosphorylated runt-related transcription factor 2 ( $\mathrm{p}$-runx2, a transcription factor involved in activation of bone related genes), and osteopontin (a mature bone extracellular matrix protein) were carried out, respectively, at days 3 and 14, as we have previously shown these to be expressed at these time points during MSC differentiation to osteoblasts. ${ }^{19}$ As before, no MSC migration was noted when spheroids were cocultured with control models (i.e., no scratch), while migration was clearly observed toward the wound activated models (i.e., scratched) (Figure 3A). With regard to MSC differentiation, no osteoblast markers were noted with spheroids coculture with fibroblast monolayers. However, there was evidence for early p-runx 2 staining at day 3 (Supplementary Figure 12) and clear expression of the mature bone marker, osteopontin, and staining at day 14 (Figure 3A). To analyze this further, spheroids were extracted from the gels (so as not to include monolayer information in the analysis), and RNA was isolated at days 1 and 3 from both control and activated osteoblast wound cocultures and analyzed via fluidigm quantitative PCR with respect to various stem cell and early osteoblast markers, including runx 2 . Results demonstrated a large increase in MSC runx 2 expression at day 3 in the activated osteoblast model, with no elevation in the control model (Figure 3B; Supplementary Figure 13). Meanwhile, chondrocyte differentiation was assessed via collagen type II immunostaining and parallel spheroid RNA isolation and analysis via fluidigm for early chondrocyte markers at day 3 . MSC migration was again evident when cocultured with wounded cocultures (Figure 4A), and evidence for collagen type II expression in spheroids was identified (Figure 4A

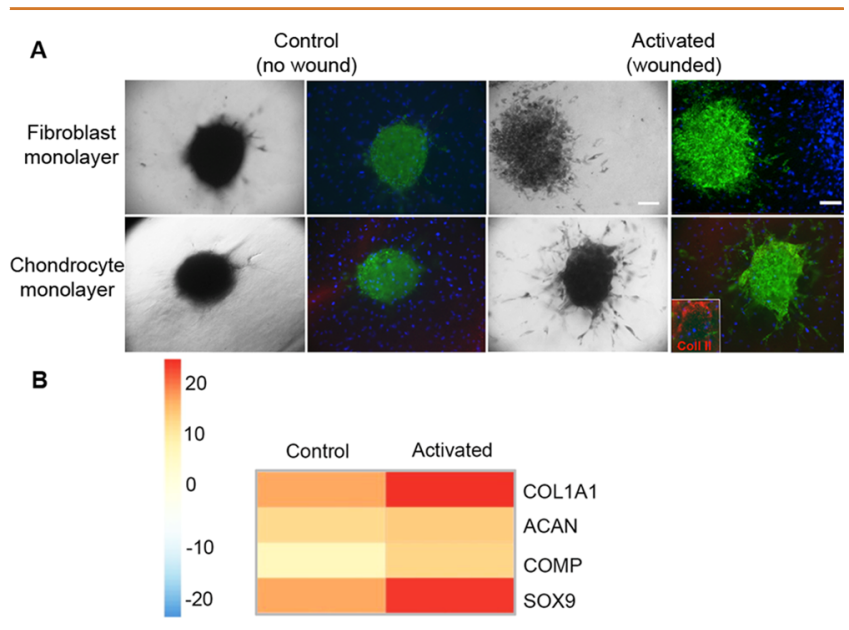

Figure 4. MSC chondrogenic differentiation during wound healing. (A) MSC migration and chondrocyte differentiation assessed at day 3 of culture, in control and activated fibroblast and chondrocyte wound models. (i) Light microscopy image of spheroids and corresponding (ii) immunofluorescent images of spheroids and monolayer cells $($ red $=$ collagen type II, green $=\mathrm{mNPs}$, blue $=$ nucleus). $10 \times$ objective, scale bar $=50 \mu \mathrm{m}$. MSC migration from the spheroids was noted in both activated models, while collagen II was identified in chondrogenic activated models only. (B) Chondrogenic differentiation markers during control and activated chondrocyte wound healing model (days 3 ). MSC spheroid RNA was harvested and analyzed via fluidigm on days 3 of coculture with both the control and activated chondrocyte wound models and expressed as a heat map. Higher expression trends of chondrogenic markers were identified in the activated day 3 model (collagen type I alpha I, aggrecan, cartilage oligomeric matrix protein and sox9). subset). Fluidigm data supported chondrocyte differentiation in spheroids, with a trend toward early chondrocyte markers (COL1A1, ACAN, COMP, and SOX9) at day 3 (Figure 4B).

IL-6 Identified as Key Paracrine Signal for MSC Migration from the Spheroid Niche. Finally we identified the MSC migratory signal released by the fibroblasts, osteoblasts, and chondrocytes during the wound healing process. Following a literature search, an initial luminex multiplex assay of five potential cytokine candidates was run on media isolated from activated and nonactivated fibroblast and osteoblast monolayers (IL-1b, IL-2, IL-6, TNF- $\alpha$, and IL12p70). The only cytokine increased in all cultures was IL-6 (Figure 5A). IL-6 was subsequently added to spheroid cultures at physiological concentrations detected in the luninex assay $(450 \mathrm{pg} / \mathrm{mL})$ to assess for MSC migration on demand; cells were noted to begin migrating from the spheroid following $12 \mathrm{~h}$ IL- 6 treatment and were clearly visible by 24 and $48 \mathrm{~h}$. MSCs in control conditions (no IL-6 treatment) remained within their spheroids (Figure 5B).

\section{CONCLUSIONS}

In conclusion, we have developed a MSC regeneration responsive, functional tissue engineered MSC bone marrow niche in vitro. The primary MSC spheroids clearly express STRO-1 and nestin and remain quiescent up to day 14 in culture. During culture they can be stimulated to transmigrate from the "niche" in response to injury and differentiate on engraftment, in particular to IL-6 migratory signals. Further work will confirm that differentiation is truly tissue specific and not generic osteochondral differentiation. This in itself is a very simple, efficient, and cost-effective model to produce and maintain. The presence of the magnetic nanoparticles not only facilitates rapid formation of spheroids in culture but also will permit cell tracking and monitoring postspheroid formation via either light microscopy or fluorescent microscopy (due to a conjugated fluorescent tag), with the potential for magnetic resonance imaging in vivo.

Such models will be invaluable for stem cell biology and in new clinical developments. For example, this model will facilitate the understanding of homing mechanisms and subsequent engraftment of MSCs in the target-injured tissue. This area of research is currently difficult to study due to the lack of robust, reproducible humanized in vitro model systems. Specifically, while integrins are known to play a key role in MSC motility (adhesion and migration), the participation of additional factors involved in transmigration such as growth factors, matrix metalloproteinases, and chemokines (e.g., CXCR4-SDF-1 axis) remains largely unknown. The unravelling of the roles of these and other factors will be crucial in enhancing MSC homing and engraftment in clinical use. Furthermore, functional nanoniches cultured in tissue plastic wells will permit drug screens to find new leads to aid regeneration. For example, testing of drugs to promote osteogenesis to aid in therapy, such as for osteoporosis, where reduction in bone density results in weakened bones.

Overall our in vitro tissue engineered MSC bone marrow niche provides the potential to study MSC regeneration response to both injury and disease conditions, thus facilitating the study of natural repair mechanisms and the processes involved in replacing diseased tissues. 

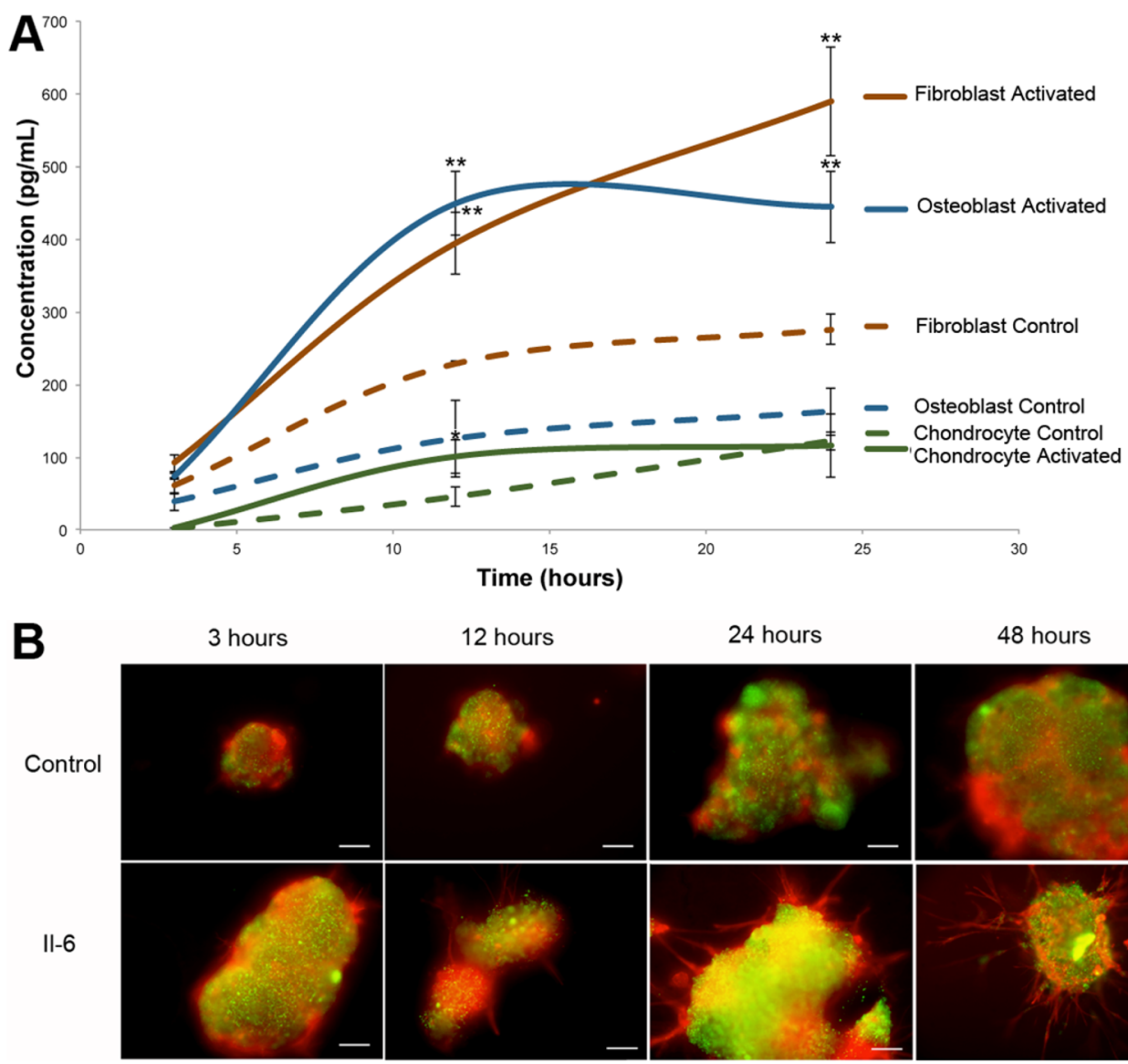

24 hours

48 hours

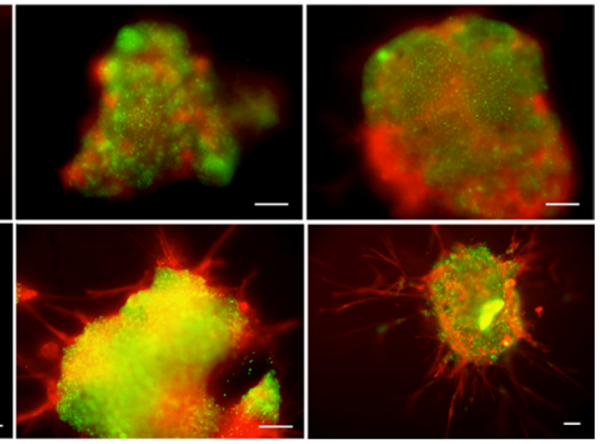

Figure 5. MSC migratory signal identified as IL-6. (A) Luminex data plot of IL-6 response from fibroblast, osteoblast, and chondrocyte control (unscratched) and activated (scratched) monolayers over $24 \mathrm{~h}$ (graph represents $n=3$, mean \pm SD; stats indicate one-way ANOVA significance $(*)$ for activated compared to corresponding control). (B) Corresponding MSC migration from spheroids in response to 450 pg/ mL IL-6 added to spheroid media.

\section{METHODS/EXPERIMENTAL}

Magnetic Nanoparticles. The mNPs employed were $200 \mathrm{~nm}$ in diameter, with an iron oxide core $\left(\mathrm{Fe}_{3} \mathrm{O}_{4}\right)$ coated in polydimethylamine, with or without a FITC fluorescent tag (green) (Chemicell, Germany).

Cell Culture. The MSCs (Promocell, Germany) were cultured in modified alpha-MEM media (10\% FBS, $2 \%$ antibiotics) (SigmaAldrich,U.K.) at $37{ }^{\circ} \mathrm{C}$ with $5 \% \mathrm{CO}_{2}$. Once confluent, the cells were trypsinized, centrifuged, resuspended in fresh media, and seeded into appropriate wells for experiments.

Monolayer Culture System. The MSCs were seeded into a 24well plate $\left(3 \times 10^{4}\right.$ cells/well $)$ and incubated overnight in $1 \mathrm{~mL}$ of media. Fresh media containing $\mathrm{mNPs}(0.1 \mathrm{mg} / \mathrm{mL})$ was then added to the cultures, and cells were incubated for a further $30 \mathrm{~min}$ in the presence of a magnetic field (Supplementary Figures 1-3). Cells were washed, trypsinized, centrifuged resuspended in media, and seeded onto sterile glass coverslips ( $13 \mathrm{~mm}$ diameter) for the appropriate incubation period (Supplementary Figures 4 and 5). Media was changed every 3 days.

Spheroid Culture System. The MSCs were seeded at a concentration of $1 \times 10^{4}$ cells/well and, following incubation with the mNPs, were trypsinized, pooled together, and centrifuged. The cells were resuspended in fresh media $(0.5 \mathrm{~mL} /$ sample $)$, and $0.5 \mathrm{~mL}$ of cell suspension was added to $4 \mathrm{~mL}$ fresh media/well (6-well plate). A single neodymium magnet $(15 \times 5 \mathrm{~mm}, 3500 \mathrm{G} / 350 \mathrm{mT}$; First4magnets, U.K.) was placed on top of the well, and the plate was incubated for the required experimental period. Cells levitated into spheroids within hours (Supplementary Figure 5). Media was changed every 3 days.
Cell Viability. After the appropriate incubation period, the cells were assessed for cell viability (LIVE/DEAD Viability/Cytotoxicity Kit, Invitrogen) by preparing $1 \mathrm{~mL}$ of fresh media containing $1 \mu \mathrm{L}$ calcein $\mathrm{AM}$ and $1 \mu \mathrm{L}$ ethidium homodimer $(1 \mathrm{~mL} / \mathrm{sample})$. This media was added to the cultures for $1 \mathrm{~h}$ at $37{ }^{\circ} \mathrm{C}$. The cells were washed three times with fresh media and analyzed immediately afterward.

Immunostaining. STRO-1/Nestin/Phosphorylated runx2/Osteopontin. Following $\mathrm{mNP}$ incubation, the cells were washed with PBS, fixed for $15 \mathrm{~min}$ with $4 \%$ paraformaldehyde/PBS, permeabilized for 5 $\min \left(10.3 \%\right.$ sucrose, $0.3 \% \mathrm{NaCl}, 0.06 \% \mathrm{MgCl}_{2}, 0.5 \%$ HEPES, and $0.5 \%$ Triton $\mathrm{X}$ in PBS), and then 1\% BSA/PBS was added to each well for 5 min. Subsequently, anti-STRO-1/antinestin/antiphosphorylated runx2/antiosteopontin (1:50 in 1\% BSA/PBS) was added for $1 \mathrm{~h}$ at $37^{\circ} \mathrm{C}$, washed well with $0.5 \%$ Tween $20 / \mathrm{PBS}$, followed by incubation with a relevant biotinylated secondary (1:50 in $1 \%$ BSA/PBS) for $1 \mathrm{~h}$ at $37{ }^{\circ} \mathrm{C}$. The cells were washed, and finally streptavidin-Texas Red (1:50 in $1 \% \mathrm{BSA} / \mathrm{PBS}$ ) was added to each well for $30 \mathrm{~min}$ at $4{ }^{\circ} \mathrm{C}$. Following washing, cells were mounted with DAPI.

$\mathrm{BrdU}$. Cells were assessed for quiescence via BrdU incorporation (Cell Proliferation Kit, GE Healthcare Life Sciences). Following mNP incubation, $6 \mathrm{~h}$ prior to fixing the cells, $1 \mathrm{mM}$ BrdU solution in DMEM was added to each sample. The cells were washed, fixed, and permeabilized using the same method as the STRO-1/Nestin immunostaining protocol. Following permeabilization, 1\% BSA/PBS was added for $10 \mathrm{~min}$. Subsequently, anti-BrdU (1:100 in DNase) was added for $2.5 \mathrm{~h}$, washed with $0.5 \%$ Tween $20 / \mathrm{PBS}$, and a secondary Texas Red (1:50 in 1\% BSA/1× PBS) was added and incubated for 1 h. The cells were washed and finally mounted with DAPI. 
Actin. Following the addition of mNPs and after the appropriate incubation period, the cells were washed, fixed, and permeabilized using the same method as the STRO-1/Nestin immunostaining protocol. Following permeabilization, 1\% BSA/PBS was added for 5 min. F-actin was stained using rhodamine-phalloidin (Invitrogen, U.K.) (1:500 in 1\% BSA/PBS) for $1 \mathrm{~h}$. The cells were washed with $0.5 \%$ Tween 20/PBS and mounted onto slides with DAPI.

All fluorescent images were taken using a Zeiss Axiovert $200 \mathrm{M}$ fluorescent microscope.

Wound Healing Assay. Collagen Spheroid Preparation. All reagents were maintained on ice prior to use. $0.5 \mathrm{~mL}$ fresh media with $0.5 \mathrm{~mL}$ 10X DMEM (First Link, U.K.) and $0.5 \mathrm{~mL}$ FBS were mixed together. $2.5 \mathrm{~mL}$ acid solubilized collagen Type I solution (First Link, U.K.) and $1 \mathrm{~mL} 0.1 \mathrm{M} \mathrm{NaOH}$ were prepared, mixed homogeneously, and then added to the FBS mixture. A dropwise addition of $0.1 \mathrm{M}$ $\mathrm{NaOH}$ was then added to the mixture to neutralize the acid solution, until pH 7 (pink color change). The suspension was then added to the appropriate wells in a 24-well plate $(0.5 \mathrm{~mL})$, and preformed spheroids were placed in the collagen gel, which was then incubated for the required experimental period. Media was changed every 3 days.

Fibroblast and Primary Osteoblast Monolayer Wound Assay. Briefly, both h-TERT (telomerase altered human dermal fibroblasts (ATCC, U.K.) $\left(1 \times 10^{5}\right.$ cells/well $)$ and primary osteoblasts $\left(5 \times 10^{4}\right.$ cells/well) (Promocell, Germany) were cultured as a confluent monolayer and left either unscratched or scratched with a P1000 pipet tip (Supplementary Figure 6). For the osteoblast monolayer culture, cells were seeded onto CellBind plates (VWR Chemicals, U.K.), and osteogenic induction media (DMEM, 10\% FBS, 2\% antibiotics, $350 \mathrm{mM}$ ascorbate-2-phosphate and $0.1 \mu \mathrm{M}$ Dexamethasone) was added every $2 / 3$ days for 10 days prior to the scratch, and collagen spheroid was added immediately after the induced scratch and monitored for the appropriate period.

RNA Extraction. RNeasy micro kit (Qiagen, U.K.) and RNase-free water were used during this experimental setup. After the appropriate incubation period, cells were lysed with $1 \mathrm{~mL}$ of TRIzol (Life Technologies, U.K.) for $10 \mathrm{~min}$ and then centrifuged for $15 \mathrm{~min}$. The supernatant was transferred and mixed thoroughly with $200 \mu \mathrm{L}$ chloroform and left for $3 \mathrm{~min}$. The mixture was centrifuged for $15 \mathrm{~min}$, the aqueous phase was removed, and glycoblue $(1 \mu \mathrm{L})$ and isopropanol $(500 \mu \mathrm{L})$ were added to the solution. The Eppendorf tubes were inverted several times and left for $10 \mathrm{~min}$, followed by centrifugation for $20 \mathrm{~min}$. The supernatant was removed leaving a blue pellet, which was vortexed with $1 \mathrm{~mL}$ ethanol $(75 \%$ aq) and then centrifuged for $5 \mathrm{~min}$. The ethanol was removed to air-dry the pellet, and water $(20 \mu \mathrm{L})$ was added to the samples, which were incubated for $10 \mathrm{~min}$. Each sample was made up to $350 \mu \mathrm{L}$ with buffer RLT and vortexed, followed by addition of $70 \%$ ethanol $(350 \mu \mathrm{L})$. This was mix thoroughly and transferred to a spin column for centrifuging for $15 \mathrm{~s}$. Buffer RW1 $(350 \mu \mathrm{L})$ was added to the spin column and centrifuged for $15 \mathrm{~s}$, and $80 \mu \mathrm{L}$ DNase I solution in buffer RDD (1:8 dilution) was added to the column for $15 \mathrm{~min} .350 \mu \mathrm{L}$ buffer RW1 was added to the column and centrifuged for 15 s. $500 \mu \mathrm{L}$ buffer RPE was added to the spin column and centrifuged for $15 \mathrm{~s}$, and $500 \mu \mathrm{L}$ of $80 \%$ ethanol was added. The column was centrifuged at full speed for $2 \mathrm{~min}$. The spin column was further centrifuged at full speed for $5 \mathrm{~min}$. The dried spin column was placed in a $1.5 \mathrm{~mL}$ Eppendorf tube, and $14 \mu \mathrm{L}$ water was added then centrifuged at full speed for $1 \mathrm{~min}$.

Analysis was conducted on confluent primary osteoblast monolayers, which were either left unscratched or scratched with a P1000 pipet tip. Cells were seeded onto CellBind plates, and osteogenic differentiation media was added for 10 days, prior to the addition of collagen spheroid implant cultures. After 24 or $72 \mathrm{~h}$, the collagen gels containing the spheroids were removed from the wells ready for RNA extraction. Six samples were pooled for each replicate $(n=3)$.

Fluidigm Analysis. The RNA was subjected to a reverse transcription using the SuperScript III Reverse Transcriptase (Invitrogen, U.K.). At all stages of the process, reactions were preformed at $4{ }^{\circ} \mathrm{C}$ unless stated. Eleven $\mu \mathrm{L}$ of each sample was added to $1 \mu \mathrm{L}$ of oligo(dT) and $1 \mu \mathrm{L} \mathrm{dNTPmix} \mathrm{and} \mathrm{then} \mathrm{heated} \mathrm{to} 65^{\circ} \mathrm{C}$ for $5 \mathrm{~min}$. A mixture containing $4 \mu \mathrm{L} 5 \times$ First Strand buffer, $1 \mu \mathrm{L} 0.1 \mathrm{M}$
DTT, $1 \mu \mathrm{L}$ RNaseOUT Recombinant RNase inhibitor, $0.5 \mu \mathrm{L}$ SuperScript III RT and $0.5 \mu \mathrm{L}$ water was prepared and added to each sample and left for $5 \mathrm{~min}$. The solution was then incubated at $50{ }^{\circ} \mathrm{C}$ for $30 \mathrm{~min}$ followed by $70{ }^{\circ} \mathrm{C}$ for $15 \mathrm{~min}$ providing cDNA. All 48 primers were pooled together $(1 \mu \mathrm{L}$ from each primer set pooled in $152 \mu \mathrm{L}$ of DNA suspension buffer). A new solution was prepared with $1.25 \mu \mathrm{L}$ of the cDNA of each sample, $2.5 \mu \mathrm{L} 2 \times$ TaqMan PreAmp Master Mix (Applied Biosystems), $0.5 \mu \mathrm{L}$ pooled primer mix, and 0.75 $\mu \mathrm{L}$ water. This was vortexed, centrifuged, and subjected to 22 thermal cycles with the following program (Table 1):

\section{Table 1}

\begin{tabular}{lllll} 
condition & hold & 22 cycles & & hold \\
temperature & $95{ }^{\circ} \mathrm{C}$ & $95{ }^{\circ} \mathrm{C}$ & $60{ }^{\circ} \mathrm{C}$ & $4{ }^{\circ} \mathrm{C}$ \\
time & $10 \mathrm{~min}$ & $15 \mathrm{~s}$ & $4 \mathrm{~min}$ & $\infty$ \\
\hline
\end{tabular}

After the 22 thermal cycles, $1.4 \mu \mathrm{L}$ water, $0.2 \mu \mathrm{L}$ Exonuclease I Reaction Buffer, and $0.4 \mu \mathrm{L}$ Exonuclease were added to each sample and vortexed, centrifuged, and incubated at $37^{\circ} \mathrm{C}$ for $30 \mathrm{~min}$ and then $80^{\circ} \mathrm{C}$ for $15 \mathrm{~min}$. After heating, $18 \mu \mathrm{L}$ of TE buffer was added to each sample. $2.7 \mu \mathrm{L}$ of the exonuclease I treated sample was added to 3.0 $\mu \mathrm{L} 2 \times$ SsoFast EvaGreen Supermix (Bio-Rad) and $0.3 \mu \mathrm{L} 20 \times$ DNA Binding Dye sample loading reagent (Fluidigm). Each mixture was vortexed and centrifuged ready to be loaded onto the chip. Additionally, $0.3 \mu \mathrm{L}$ of each individual primer set was added to 3 $\mu \mathrm{L} 2 \times$ assay loading reagent and $2.7 \mu \mathrm{L} 1 \times$ DNA suspension buffer that was vortexed and centrifuged, ready for loading on the chip and run on the Fluidigm Biomark HD system. A 48.48 Dynamic array IFC was used during this analysis.

Luminex 100 Analysis. As a confluent monolayer, either primary osteoblasts or h-TERTs were used and left either unscratched or scratched using a P1000 pipet tip. For the osteoblast monolayer culture, cells were seeded as a confluent monolayer onto CellBind plates, and osteogenic differentiation media was added every $2 / 3$ days for 10 days and then either scratched or left unscratched. While for the h-TERTs monolayer, cells were seeded as a confluent monolayer and left for $24 \mathrm{~h}$, then either scratched or left unscratched.

Fresh media was added, and the supernatant was removed after 3 , 12, 24, and $72 \mathrm{~h}$, and the Bio-Rad Bio-Plex Pro cytokine assay protocol was used to analyze the cytokines. All solutions used were prepared from the Bio-Rad Pro Human Cytokine Group I, 5-plex assay kit except for the fresh media. Each supernatant was centrifuged at $1000 \mathrm{~g}$ at $4{ }^{\circ} \mathrm{C}$ for $15 \mathrm{~min}$, and the media was removed, ready for processing. The standard was reconstituted with fresh media, and 8 serial dilutions (1:4) were followed with fresh media to create a standard curve as described in the protocol. A control blank was prepared with fresh media. Each sample was additionally diluted (1:4) with fresh media. Stock solutions $(10 \times)$ of conjugated coupled magnetic beads were pooled together with assay buffer to make a single $1 \times$ solution. The plate was prewetted with $100 \mu \mathrm{L}$ assay buffer, which was removed, and $50 \mu \mathrm{L}$ of the $1 \times$ magnetic beads solution was added. Prior to the removal of each solution from the plate, a magnetic plate was applied to retain the beads within the wells. The solution was removed, and the plate was washed twice with $100 \mu \mathrm{L}$ of wash buffer. The standards, samples, and blanks $(50 \mu \mathrm{L})$ were added to the appropriate wells and left covered, shaking at room temperature for $30 \mathrm{~min}$. After the appropriate incubation period, the solution was removed and the wells were washed thrice with wash buffer $(100 \mu \mathrm{L})$. Stock solutions $(10 \times)$ of detection antibodies were mixed together and diluted to create a $1 \times$ solution with detection antibody diluent. The $1 \times$ solution was added to the wells $(25 \mu \mathrm{L})$ and left covered, shaking at room temperature for $30 \mathrm{~min}$. After the appropriate incubation period, the solution was removed, and the wells were washed thrice with wash buffer $(100 \mu \mathrm{L})$. Stock solution $(100 \times)$ of streptavidin-PE was diluted to create a $1 \times$ solution with assay buffer. The $1 \times$ solution was added to the wells (50 $\mu \mathrm{L}$ ) and left covered, shaking at room temperature for $10 \mathrm{~min}$. After the appropriate incubation period, the solution was removed, and the 
Table 2. Primer Sequences

\begin{tabular}{|c|c|c|c|c|c|c|c|}
\hline Primer Name & $\begin{array}{l}\text { Forward Sequence }\left(5^{\prime}->3^{\prime}\right) \\
\text { Back Sequence }\left(5^{\prime} \rightarrow 3^{\prime}\right)\end{array}$ & Primer Name & $\begin{array}{l}\text { Forward Sequence }\left(5^{\prime} \rightarrow 3^{\prime}\right) \\
\text { Back Sequence }\left(5^{\prime} \rightarrow 3^{\prime}\right)\end{array}$ & Primer Name & $\begin{array}{l}\text { Forward Sequence }\left(5^{\prime} \text { - }^{\prime} 3^{\prime}\right) \\
\text { Back Sequence }\left(5^{\prime}>3^{\prime}\right)\end{array}$ & Primer Name & $\begin{array}{l}\text { Forward Sequence }\left(5^{\prime}->3^{\prime}\right) \\
\text { Back Sequence }\left(5^{\prime} \rightarrow 3^{\prime}\right)\end{array}$ \\
\hline B-Actin & $\begin{array}{l}\text { GTGGGCCGCCCTAGGCACCAG } \\
\text { CACTTTGATGTCACGCACGATTTC }\end{array}$ & BMPR1B & $\begin{array}{l}\text { GGTTCAGACTTCTGCTGATTCAT } \\
\text { CGCAAAAGCATGTTATCAAGG }\end{array}$ & RUNX1T1 & $\begin{array}{l}\text { ATCACAACAGAGAGGGCCAA } \\
\text { CTGCAGGTTTCACTCGCTTT }\end{array}$ & CathepsinB & $\begin{array}{l}\text { TGTGTATTCGGACTTCCTGC } \\
\text { TTAAAGAAGCCATTGTCACCC }\end{array}$ \\
\hline RUNX2 & $\begin{array}{l}\text { CAGCAGCAGCAACAGCAG } \\
\text { GGCGATGATCTCCACCAT }\end{array}$ & \begin{tabular}{|l|} 
BMP2 \\
\end{tabular} & $\begin{array}{l}\text { CTTCTAGCGTTGCTGCTTCC } \\
\text { AACTCGCTCAGGACCTCGT }\end{array}$ & SMURF1 & $\begin{array}{l}\text { ATGCAGTTCGTGGCCAGATA } \\
\text { CAGGCCCGGAGTCTTCATAC }\end{array}$ & CathepsinD & $\begin{array}{l}\text { GGTGCTCAAGAACTACATGG } \\
\text { ATTCTTCACGTAGGTGCTGG }\end{array}$ \\
\hline ACVR1A & $\begin{array}{l}\text { GCCAAGGGGACTGGTGTAAC } \\
\text { GAGAATAATGAGGCCAACCTCCA }\end{array}$ & BMP2 & $\begin{array}{l}\text { AGACCTGTATCGCAGGCACT } \\
\text { CCACTCGTTTCTGGTAGTTCTTCC }\end{array}$ & SMURF2 & $\begin{array}{l}\text { GACAGGATCCTCTCGAGTGC } \\
\text { AGCTTTCATAGGGTGGAATGTCT }\end{array}$ & CathepsinG & $\begin{array}{l}\text { AACAGATACACTCCGAGAGG } \\
\text { ACGACTTTCCATAGGAGACG }\end{array}$ \\
\hline SMAD1 & $\begin{array}{l}\text { GCTGCTCTCCAATGTTAACCG } \\
\text { CACTAAGGCATTCGGCATACAC }\end{array}$ & BMPR2 & $\begin{array}{l}\text { AGCCTCTCACACCCACTCC } \\
\text { GCAGAACAACCGTGAGAGG }\end{array}$ & INHBA & $\begin{array}{l}\text { AAGTCGGGGAGAACGGGTAT } \\
\text { GGTCACTGCCTTCCTTGGAA }\end{array}$ & CathepsinL & $\begin{array}{l}\text { GACTCTGAGGAATCCTATCC } \\
\text { CTTAGGGATGTCCACAAAGC }\end{array}$ \\
\hline SMAD2 & $\begin{array}{l}\text { CCACGGTAGAAATGACAAGAAGG } \\
\text { GATTACAATTGGGGCTCTGCAC }\end{array}$ & ACVRIB & $\begin{array}{l}\text { GACATTGCCCCGAATCAGAGG } \\
\text { GCCCGAGGGCATAAATATCAGC }\end{array}$ & ACVR2A & $\begin{array}{l}\text { ACCATGGCTAGAGGATTGGC } \\
\text { GCCAACCCAAAGTCAGCAAT }\end{array}$ & CathepsinS & $\begin{array}{l}\text { GCGTCATCCTTCTTTCTTCC } \\
\text { CCAGCTGTTTTTCACAAGCC }\end{array}$ \\
\hline SMAD3 & $\begin{array}{l}\text { GTCTGCGTGAATCCCTACCAC } \\
\text { GGGATGGAATGGCTGTAGTCG }\end{array}$ & BMP4 & $\begin{array}{l}\text { CAGCACTGGTCTTGAGTATCCT } \\
\text { AGCAGAGTTTTCACTGGTCCC }\end{array}$ & ACVR2B & $\begin{array}{l}\text { CTGCAACGAACGCTTCACTC } \\
\text { CAGGACGATGAGGGAAAGGC }\end{array}$ & GAPDH & $\begin{array}{l}\text { TCAAGGCTGAGAACGGGAA } \\
\text { TGGGTGGCAGTGATGGCA }\end{array}$ \\
\hline ENOX2 & $\begin{array}{l}\text { GAGCTGGAGGGAACCTGATTT } \\
\text { CACTGGCACTACCAAACTGCA }\end{array}$ & CYCR & $\begin{array}{l}\text { ACTGCGGGAAGGTCTCTACTT } \\
\text { GGGTGCCATCGTCAAACTCTA }\end{array}$ & RNF20 & $\begin{array}{l}\text { GGTGTCTCTTCAACGGAGGAA } \\
\text { TAGTGAGGCATCATCAGTGGC }\end{array}$ & & \\
\hline SMAD4 & $\begin{array}{l}\text { GGGTCAACTCTCCAATGTCCAC } \\
\text { GTCACTAAGGCACCTGACCC }\end{array}$ & BMP7 & $\begin{array}{l}\text { CAGGCCTGTAAGAAGCACGA } \\
\text { TGGTTGGTGGCGTTCATGTA }\end{array}$ & TGFB 1 & $\begin{array}{l}\text { CGACTCGCCAGAGTGGTTATC } \\
\text { GTTATCCCTGCTGTCACAGGAG }\end{array}$ & & \\
\hline SMAD5 & $\begin{array}{l}\text { TGGGTCAAGATAATTCCCAGCCT } \\
\text { GGCTCTTCATAGGCAACAGGC }\end{array}$ & BMP10 & $\begin{array}{l}\text { ACCCACCAGAGTACATGTTGG } \\
\text { GCCCATTAAAACTGACCGGC }\end{array}$ & TGFBRI & $\begin{array}{l}\text { CGTTCGTGGTTCCGTGAGG } \\
\text { TAATCTGACACCAACCAGAGCTG }\end{array}$ & & \\
\hline SMAD6 & $\begin{array}{l}\text { CTCCCTACTCTCGGCTGTCT } \\
\text { AGAATTCACCCGGAGCAGTG }\end{array}$ & Nestin & $\begin{array}{l}\text { GCTCAGGTCCTGGAAGGTC } \\
\text { AAGCTGAGGGAAGTCTTGGA }\end{array}$ & TRAF6 & $\begin{array}{l}\text { CGCACTAGAACGAGCAAGTGA } \\
\text { GCCACACAGCAGTCACTTTCA }\end{array}$ & & \\
\hline SMAD7 & $\begin{array}{l}\text { CCATCACCTTAGCCGACTCT } \\
\text { CCAGGGGCCAGATAATTCGT }\end{array}$ & CD63 & $\begin{array}{l}\text { CCCTTGGAATTGCTTTTGTT } \\
\text { TATTCCACTCCCCCAGATGA }\end{array}$ & SNAIL2 & $\begin{array}{l}\text { TCCTTCCTGGTCAAGAAGCA } \\
\text { GGTATGACAGGCATGGAGTA }\end{array}$ & & \\
\hline SMAD9 & $\begin{array}{l}\text { CTTATCATGCCACAGAAGCCTCT } \\
\text { GCTCCTCGTAACAAACTGGTCG }\end{array}$ & ALCAM & $\begin{array}{l}\text { TTCCAGTCCCTCTACTCAGAGC } \\
\text { GCTAAGAAGGACTCGCAGGA }\end{array}$ & Vimentin & $\begin{array}{l}\text { GGAGAAATTGCAGGAGGAGA } \\
\text { TGCGTTCAAGGTCAAGACGT }\end{array}$ & & \\
\hline BMPR1A & $\begin{array}{l}\text { ACGCCGGACAATAGAATGTTGTC } \\
\text { GAGCAAAACCAGCCATCGAATG }\end{array}$ & Osterix & $\begin{array}{l}\text { TGGGCTCCCAACACTATTTC } \\
\text { GGGAAGACTGAAGCCTGGA }\end{array}$ & IL-08 & $\begin{array}{l}\text { GTGTGAAGGTGCAGTTTTGCC } \\
\text { GTGGTCCACTCTCAATCACTC }\end{array}$ & & \\
\hline TWY1 & $\begin{array}{l}\text { ATTGTCATCAAGACGCAGGGC } \\
\text { GTTGCGAATCCCTTCGCTGTT }\end{array}$ & UBE2D2 & $\begin{array}{l}\text { CCATGGCTCTGAAGAGAATCC } \\
\text { GATAGGGACTGTCATTTGGCC }\end{array}$ & $\mathrm{B} 2 \mathrm{M}$ & $\begin{array}{l}\text { TTGTCTTTCAGCAAGGACTGG } \\
\text { ATGCGGCATCTTCAAACCTCC }\end{array}$ & & \\
\hline
\end{tabular}

wells were washed thrice with wash buffer $(100 \mu \mathrm{L})$. The beads were resuspended with assay buffer $(125 \mu \mathrm{L})$ ready for analysis.

Primer Sequences (Fluidigm). Primer sequences can be found in Table 2.

\section{ASSOCIATED CONTENT}

\section{S Supporting Information}

The Supporting Information is available free of charge on the ACS Publications website at DOI: 10.1021/acsnano.6b02841.

Experimental details and data (PDF)

\section{AUTHOR INFORMATION}

\section{Corresponding Author}

*E-mail: Catherine.berry@glasgow.ac.uk.

\section{Notes}

The authors declare no competing financial interest.

\section{ACKNOWLEDGMENTS}

All authors with to acknowledge funding from Research Councils of the United Kingdom (RCUK) BBSRC; BB/ L008661/1 and EPSRC; EP/F500424/1; and N. Lewis for schematics.

\section{REFERENCES}

(1) Lane, S. W.; Williams, D. A.; Watt, F. M. Modulating the Stem Cell Niche for Tissue Regeneration. Nat. Biotechnol. 2014, 32, 795803.

(2) Voog, J.; Jones, D. L. Stem Cells and the Niche: a Dynamic Duo. Cell Stem Cell 2010, 6, 103-115.

(3) Laine, S. K.; Hentunen, T.; Laitala-Leinonen, T. Do MicroRNAs Regulate Bone Marrow Stem Cell Niche Physiology? Gene 2012, 497, $1-9$.

(4) Ehninger, A.; Trumpp, A. The Bone Marrow Stem Cell Niche Grows Up: Mesenchymal Stem Cells and Macrophages Move. J. Exp. Med. 2011, 208, 421-428.

(5) Park, D.; Spencer, J. A.; Koh, B. I.; Kobayashi, T.; Fujisaki, J.; Clemens, T. L.; Lin, C. P.; Kronenberg, H. M.; Scadden, D. T. Endogenous Bone Marrow MSCs are Dynamic, Fate-Restricted Participants in Bone Maintenance and Regeneration. Cell Stem Cell 2012, 10, 259-272.

(6) Dalby, M. J.; Gadegaard, N.; Oreffo, R. O. C. Harnessing Nanotopography and Integrin-Matrix Interactions to Influence Stem Cell Fate. Nat. Mater. 2014, 13, 558-569.

(7) Murphy, W. L.; McDevitt, T. C.; Engler, A. J. Materials as Stem Cell Regulators. Nat. Mater. 2014, 13, 547-557.

(8) McMurray, R. J.; Gadegaard, N.; Tsimbouri, P. M.; Burgess, K. V.; McNamara, L. E.; Tare, R.; Murawski, K.; Kingham, E.; Oreffo, R. O.; Dalby, M. J. Nanoscale Surfaces for the Long-Term Maintenance of Mesenchymal Stem Cell Phenotype and Multipotency. Nat. Mater. 2011, 10, 637-644. 
(9) Di Maggio, N.; Piccinini, E.; Jaworski, M.; Trumpp, A.; Wendt, D. J.; Martin, I. Toward Modeling the Bone Marrow Niche using Scaffold-Based 3D Culture Systems. Biomaterials 2011, 32, 321-329.

(10) Bartosh, T. J.; Ylostalo, J. H.; Mohammadipoor, A.; Bazhanov, N.; Coble, K.; Claypool, K.; Lee, R. H.; Choi, H.; Prockop, D. J. Aggregation of Human Mesenchymal Stromal Cells (MSCs) into 3D Spheroids Enhances their Antiinflammatory Properties. Proc. Natl. Acad. Sci. U. S. A. 2010, 107, 13724.

(11) Souza, G. R.; Molina, J. R.; Raphael, R. M.; Ozawa, M. G.; Stark, D. J.; Levin, C. S.; Bronk, L. F.; Ananta, J. S.; Mandelin, J.; Georgescu, M. M.; et al. Three-Dimensional Tissue Culture based on Magnetic Cell Levitation. Nat. Nanotechnol. 2010, 5, 291-296.

(12) Matsuoka, Y.; Nakatsuka, R.; Sumide, K.; Kawamura, H.; Takahashi, M.; Fujioka, T.; Uemura, Y.; Asano, H.; Sasaki, Y.; Inoue, M.; et al. Prospectively Isolated Human Bone Marrow Cell-Derived MSCs Support Primitive Human CD34-Negative Hematopoietic Stem Cells. Stem Cells 2015, 33, 1554-1565.

(13) Pinho, S.; Lacombe, J.; Hanoun, M.; Mizoguchi, T.; Bruns, I.; Kunisaki, Y.; Frenette, P. S. PDGFR $\alpha$ and CD51 Mark Human Nestin + Sphere-Forming Mesenchymal Stem Cells Capable of Hematopoietic Progenitor Cell Expansion. J. Exp. Med. 2013, 210, 1351-1367.

(14) Engler, A. J.; Sen, S.; Sweeney, H. L.; Discher, D. E. Matrix Elasticity Directs Stem Cell Lineage Specification. Cell 2006, 126, 677-689.

(15) Mendez-Ferrer, S.; Michurina, T. V.; Ferraro, F.; Mazloom, A. R.; Macarthur, B. D.; Lira, S. A.; Scadden, D. T.; Ma'ayan, A.; Enikolopov, G. N.; Frenette, P. S. Mesenchymal and Haematopoietic Stem Cells form a Unique Bone Marrow Niche. Nature 2010, 466, 829-834.

(16) Bianco, P. Bone and the Hematopoietic Niche: a Tale of Two Stem Cells. Blood 2011, 117, 5281-5288.

(17) Smith, C.-A. M.; de la Fuente, J.; Pelaz, B.; Furlani, E. P.; Mullin, M.; Berry, C. C. The Effect of Static Magnetic Fields and Tat Peptides on Cellular and Nuclear Uptake of Magnetic Nanoparticles. Biomaterials 2010, 31, 4392-4400.

(18) Dejardin, T.; de la Fuente, J.; del Pino, P.; Furlani, E. P.; Mullin, M.; Smith, C.-A.; Berry, C. C. Influence of both a Static Magnetic Field and Penetratin on Magnetic Nanoparticle Delivery into Fibroblasts. Nanomedicine 2011, 6, 1719-1731.

(19) Yang, J.; McNamara, L. E.; Gadegaard, N.; Alakpa, E. V.; Burgess, K. V.; Meek, R. M.; Dalby, M. J. Nanotopographical Induction of Osteogenesis through Adhesion, Bone Morphogenic Protein Cosignaling, and Regulation of MicroRNAs. ACS Nano 2014, 8, 9941-9953. 